\title{
Development of the Italian version of the High-Activity Arthroplasty Score (HAAS-I) following hip and knee total arthroplasty: cross-cultural adaptation, reliability, validity and sensitivity to change
}

Marco Monticone ${ }^{1}$, Antonio Capone ${ }^{2}$, Luca Frigau ${ }^{3}$, Giuseppe Marongiư², Paola Abelli', Francesco Mola ${ }^{3}$, Nicola Maffulli, ${ }^{5,6^{*}}$ (D) and Calogero Foti ${ }^{7}$

\begin{abstract}
Background: The number of physically active individuals who develop knee and hip arthritis and who undergo arthroplasties of these joints ie ever increasing. It has become necessary to develop evaluation scales which address the specific issues raised by such individuals. The High Activity Arthroplasty Score is one such scales, originally developed in English.

Methods: The HAAS-I was developed by means of forward-backward translation, a final review by an expert committee and a test of the pre-final version to establish its correspondence with the original English version. The psychometric testing included reliability by means of internal consistency (Cronbach's alpha) and test-retest reliability (intraclass correlation coefficients) and construct validity by Pearson's correlations with a pain intensity numerical rating scale (NRS), the Western Ontario and McMaster University index (WOMAC, for THA subjects), the Knee injury and Osteoarthritis Outcome Scale (KOOS; for TKA subjects) and the Short-Form 36 Health Survey (SF-36).

Results: The questionnaire was administered to 67 subjects with THA and 61 with TKA and proved to be acceptable. The questionnaire showed good internal consistency (0.85 for THA and 0.91 for TKA) and a high level of test-retest reliability $(\mathrm{ICC}=0.97$ with $95 \% \mathrm{Cl} 0.95-0.98$ for THA; ICC $=0.95$ with $95 \% \mathrm{Cl} 0.92-0.98$ for TKA). There was a moderate correlation between the HAAS-I and NRS $(r=-0.40)$, there was a high correlation between the HAAS-I and WOMAC $(r=-0.68)$ and there were moderate to high correlations between the HAAS-I and SF-36 subscales $(r=0.34$ to 0.63$)$ for THA. There was a moderate correlation between the HAAS-I and NRS $(r=-0.77)$; there was a high correlation between the HAAS-I and KOOS subscales ( $r=-0.79$ to $r=-0.91$ ); and there were low correlations between the HAAS-I and SF-36 subscales ( $r=0.01$ to 0.29 ) for TKA.
\end{abstract}

Conclusions: The HAAS-I was successfully translated into Italian and proved to have good psychometric properties that replicated the results of existing versions. Its use is recommended for clinical and research purposes.

Keywords: HAAS, Cross-cultural adaptation, Italian validation, Total hip arthroplasty, Total knee arthroplasty

\footnotetext{
* Correspondence: n.maffulli@qmul.ac.uk

${ }^{5}$ Department of Musculoskeletal Disorders, University of Salerno School of

Medicine and Surgery, Salerno, Italy

${ }^{6}$ Centre for Sports and Exercise Medicine, Barts and The London School of

Medicine and Dentistry, Mile End Hospital, 275 Bancroft Road, London, UK

Full list of author information is available at the end of the article
} 


\section{Background}

The hip and the knee are two of the most common joints which develop primary osteoarthritis, which is mainly characterized by osteophyte formation, bone remodeling and subchondral sclerosis [1]. Osteoarthritis develops gradually over several years and, as it progresses, produces hip and knee dysfunction with reduced range of motion, muscle weakness and impaired proprioception, associated with limitations during walking, activities of daily living and working activities [2].

Orthopaedic surgery has rapidly developed over the last 20-30 years, and total hip and knee arthroplasty (THA and TKA, respectively) have now become the treatment of choice for subjects with intractable pain and severe disability from hip and knee OA. The number of THA in Italy increased 32\% between 1999 and 2005, with about 50,000 new operations every year [3]. The number of subjects discharged after TKA in Italy significantly increased from 26,793 to 44,119 between 2001 and 2005, and surgical TKA revisions increased from 1166 to 2309 [3].

With such a high burden, it is of great importance to apply evidence-based, validated and comprehensive outcome measures to help clinicians to quantify and improve interventions. As supported by most researchers, a number of reliable and valid measures are available to assess functional outcomes following hip and knee arthroplasty, such as the Tegner and Lysholm score, the Oxford Knee Score (OKS), the Knee Society Clinical Rating System, the Harris Hip Score (HHS), the Merle d'Aubigne Hip score and the Western Ontario and McMaster Universities Osteoarthritis Index [4-8].

However, based on the good results of contemporary arthroplasty and the need to assess the performance in younger ages and high physical demands subjects, the High-Activity Arthroplasty Score (HAAS) was specifically developed to detect subtle variations in functional ability after lower limb arthroplasty [9]. It is a selfadministered tool published in 2010 in English; it includes four domains (i.e. running, walking, stair climbing and overall activity), with each domain consisting of one item. Despite different levels of answers, a points system was applied to each option of each item, with a higher score indicating higher functional ability; the totals deriving from each domain are collected separately producing a total score, ranging from 0 (minimum) and 18 points (maximum in functional ability) [9].

To the authors' knowledge, the HAAS has been cross-culturally adapted only in French [10]. However, non-English adaptations are of great interest as they contribute to investigate the psychometric properties of the original form of a scale, allowing comparison of results and investigating functional status across different people and countries.

\section{Purpose}

A validation study of a translated form of the HAAS was never conducted in an Italian population. As this represented a limit for clinicians and researchers of our country to share validated outcomes, the aim of this study was to describe translation, cultural adaptation and validation (internal consistency, reproducibility, validity and sensitivity to change) of the Italian version of the HAAS in adult subjects after hip and knee arthroplasty.

\section{Methods}

This cross-sectional study was approved by the Local Ethics Committee of the University of Cagliari and conducted in accordance with ethical and humane principles of research. Written consent to participate was obtained from all participants.

\section{Subjects}

The study involved outpatients attending the Orthopaedics Unit at the Marino Hospital in Cagliari and the Physical Medicine and Rehabilitation Unit at the Tor Vergata Hospital in Rome between May 2016 and June 2017. The inclusion criteria were interventions for primary uncemented THA/TKA because of primary osteoarthritis 6 months before, adult age of $<65$ years, and fluency in Italian; the exclusion criteria were cognitive impairment (i.e. Mini Mental State Examination of <24) and neurological, heart and lung co-morbidities; subjects with previous lower limb surgery, infection, fracture, osteonecrosis or malignancy and systemic or neuromuscular diseases were also excluded.

Those subjects satisfying the inclusion criteria were asked to sign a written informed consent. Once the patients had given their approval to participate to the study, their demographic and clinical characteristics were recorded by research assistants.

\section{Cross-cultural adaptation}

Adaptation of the HAAS was performed in accordance with the protocol issued by the American Association of Orthopaedic Surgeon Outcomes Committee [11]. Further, principles of good practice for the translation and cultural adaptation process for patient-reported outcomes (PRO) measures based on the report of the ISPOR task force were taken into account [12].

\section{Step 1: translation into Italian}

The items taken from the original scale were translated into Italian with the aim of retaining the concepts of the original while using culturally and clinically fitting expressions. Two translations were made independently by two Italian professional translators experienced in the 
PRO field. The translators were given a clear explanation of the concepts in the HAAS, to capture the conceptual meaning of the items. Keeping the language colloquial and compatible with a reading age of 12 years, discrepancies between the translators were resolved by means of reconciliation between them; step 1 ended when a common adaptation was agreed.

\section{Step 2: back-translation into English}

Two bilingual translators whose mother tongue was English independently back-translated the initial translation. The principal investigator (MM) reviewed these translations and, with the help of the back-translators, made sure that the Italian version reflected the same item content as the original version and was conceptually equivalent.

\section{Step 3: expert committee}

To harmonize the adaptation process, the translations were submitted to a bilingual committee of clinicians, methodologists and the translators, chaired by the principal investigator. To identify any discrepancies or mistakes, the committee explored the semantic, idiomatic and conceptual equivalence of the items and answers. This phase ended when a pre-final version was agreed.

\section{Step 4: test of the pre-final version}

This was performed to assess the level of understandability and cognitive equivalence of the translation, to highlight any items that may be inappropriate at a conceptual level and to identify any other issues that cause confusion. Cognitive interviews were therefore conducted by a trained psychologist by administering the HAAS to 10 patients with THA/TKA. The principal investigator and the Expert Committee reviewed the results from cognitive debriefing with the aim of identifying any modification necessary for improvement of the Italian form.

\section{Sample size}

Sample size was based on the "rule of 10 " patients per item [13].

\section{Scale properties \\ Feasibility}

The time needed to answer the questionnaire was recorded. The subjects were asked about any problems they encountered, and the data were checked for missing or multiple responses.

\section{Floor/ceiling effects}

Descriptive statistics were calculated to identify floor/ ceiling effects, which were considered to be present when $>15 \%$ of the subjects obtained the lowest or highest possible scores [13].

\section{Reliability}

Internal consistency (Cronbach's alpha, with values of $>0.70$ being considered acceptable) and test-retest reliability (intraclass correlation coefficient: ICC 2,1, with good and excellent reliability respectively indicated by values of $0.70-0.85$ and $>0.85$ ) [13] were investigated. The test-retest interval was 10 days.

\section{Content validity}

For the purpose of content validation, subjects were asked to report their perceptions of the aim of the measurement (question: "Do you think the aim of this questionnaire is to investigate high-intensity activities?"), the target population ("Do you think the items described here may be related to status?"), relevance ("Do you think these items are relevant to evaluating your highintensity activities?") and completeness ("Do you think that the items comprehensively reflect highintensity activities?"). The hypotheses were considered acceptable if the percentage of affirmative answers was $>90 \%$ [13].

\section{Construct validity}

For construct validation [13], it was hypothesized a priori the HAAS would achieve moderate to high correlations with: (a) disability, the Italian version of Western Ontario and McMaster University index (WOMAC) for THA [14] and the Italian version of the Knee injury and Osteoarthritis Outcome Scale (KOOS) for TKA [15]; (b) pain intensity, the 0-10 Numerical Rating Scale (NRS) [16]; and (c) quality of life, the Italian version of the Short-Form Health Survey (SF-36) [17]. The correlations with these measures were expected to be moderate. Pearson's correlations were interpreted as follows: $r<0.30$ as low, $0.30<r<0.60$ as moderate and $r>0.60$ as high.

\section{Sensitivity to change}

It was estimated by means of the minimum detectable change (MDC) calculated by multiplying the standard error of the measurements (SEM) by the $z$-score associated with the desired level of confidence ( $95 \%$ in our case) and the square root of 2, which reflects the additional uncertainty introduced by using difference scores based on measurements made at two time points (in our case on days 1 and 10). The SEM was estimated using the formula: $\operatorname{SEM}=\operatorname{SD}\left[(1-R)^{1 / 2}\right]$, where $\mathrm{SD}$ is the baseline standard deviation of the measurements and $R$ the testretest reliability coefficient [13]. 


\section{Measures}

- WOMAC: It is a multidimensional scale of 24 items grouped into three subscales: physical function (17 items), pain (5 items) and stiffness (2 items); we used the 3.1 Likert version that allows five response levels for each item (scored 0-4) representing different degrees of intensity (none, mild, moderate, severe, or extreme). The data for each subscale are standardized to a range of $0-100$, where 0 is the best and 100 the worst health status [14].

- KOOS: It has five subscales: Pain, Symptoms, Activities of Daily Living, Sport and Recreation and Knee-related Quality of Life. A 5-point Likert scale ranging from 0 (no problems) to 4 (extreme problems) is used to score each item, and the raw scores of each subscale are separately transformed into a 0-100 scale with 0 indicating the worst problems and 100 indicating no problems [15].

- NRS: This is an 11-point rating scale ranging from 0 (no pain at all) to 10 (the worst imaginable pain) [16]. Patients were asked to evaluate the pain they felt in the last week.

- SF-36: This is assessed using the Italian version of the self-report Short-Form Health Survey (SF-36).

The eight domain scores (Physical Functioning, Physical Role, Physical Pain, General Health, Vitality, Social Activities, Emotional Role and Mental Health) were calculated on the basis of the Italian User's Manual, with 0 representing the worst perceived QoL and 100 the best perceived QoL [17].

The analyses were made using the Italian version of SPSS 23.0 software.

\section{Results}

\section{Subjects}

The study involved 67 subjects with THA and 61 with TKA. There were 10 female (6.7\%) patients who underwent a THA and 29 female patients who underwent a TKA (47.5\%) with a mean age of $56.4 \pm 6.8$ years (range 43-65) for the first group and a mean age of $54.5 \pm 6.1$ years (range 45-64) for the second group. The median duration of complaints before intervention was 56 months (range 6-80) for THA and 53 months (range 6-48) for TKA. Their mean body mass index was $25.8 \pm 3.2$ for THA and $27.2 \pm 5.4$ for TKA. Table 1 shows socio-demographic characteristics.

\section{Translation and cross-cultural adaptation}

The translation procedure took 1 month to reach a culturally adapted version, and all the items were easily forward and back-translated; no difficulties were evidenced during the review of the back translations. The correctness of the process, the content of the items and the concepts
Table 1 General characteristics of subjects with total hip arthroplasty (THA, $n=67)$ and total knee arthroplasty (TKA) $(n=61)$

\begin{tabular}{|c|c|c|c|c|}
\hline \multirow[b]{2}{*}{ Variable } & \multicolumn{2}{|c|}{ THA } & \multicolumn{2}{|c|}{ TKA } \\
\hline & $N$ & $\%$ & $N$ & $\%$ \\
\hline \multicolumn{5}{|l|}{ Marital status } \\
\hline Unmarried & 17 & 25.4 & 12 & 19.7 \\
\hline Married & 50 & 74.6 & 49 & 80.3 \\
\hline \multicolumn{5}{|l|}{ Employment } \\
\hline Unemployed & 13 & 19.4 & 4 & 6.6 \\
\hline Employee & 21 & 31.3 & 27 & 44.3 \\
\hline Self-employed & 1 & 1.5 & 4 & 6.6 \\
\hline Retired & 21 & 31.3 & 14 & 23.0 \\
\hline Housewife & 10 & 16.4 & 12 & 19.7 \\
\hline \multicolumn{5}{|l|}{ Education } \\
\hline Elementary school & 2 & 3.0 & 5 & 8.2 \\
\hline Middle school & 28 & 41.8 & 26 & 42.6 \\
\hline Upper school & 27 & 40.3 & 24 & 39.3 \\
\hline University & 10 & 14.9 & 6 & 9.8 \\
\hline \multicolumn{5}{|l|}{ Smoking } \\
\hline Yes & 13 & 19.4 & 15 & 24.6 \\
\hline No & 54 & 80.6 & 46 & 75.4 \\
\hline \multicolumn{5}{|l|}{ Use of drugs } \\
\hline Antidepressants & 5 & 7.5 & 7 & 11.5 \\
\hline Analgesics & 15 & 22.4 & 15 & 24.6 \\
\hline Muscle relaxants & 5 & 7.5 & 4 & 6.6 \\
\hline NSAIDs & 7 & 10.4 & 3 & 4.9 \\
\hline None & 35 & 52.2 & 32 & 52.5 \\
\hline \multicolumn{5}{|l|}{ Comorbidities (principal) } \\
\hline Hypertension & 15 & 22.4 & 12 & 19.7 \\
\hline Non-insulin dependent diabetes mellitus & 2 & 3.0 & 2 & 3.3 \\
\hline Heart disease & 4 & 6.0 & 5 & 8.2 \\
\hline Gastro-enteric disease & 4 & 6.0 & 6 & 9.8 \\
\hline Respiratory disease & 8 & 11.9 & 6 & 9.8 \\
\hline None & 34 & 50.7 & 30 & 49.2 \\
\hline
\end{tabular}

NSAIDs non-steroidal anti-inflammatory drugs

expressed were confirmed by the experts. The cognitive interviews confirmed the comprehensibility and the cognitive equivalence of the translation; no other issues causing confusion were pointed out. Finally, the principal investigator and the Expert Committee confirmed the work performed.

The HAAS-I is reproduced in the Appendix.

\section{Psychometric scale properties Acceptability}

All of the questions were well accepted. The questionnaire was completed in $1.8 \pm 1.3 \mathrm{~min}$ for THA and $1.6 \pm 0.5 \mathrm{~min}$ for TKA. No missing responses or multiple answers were found. There were no problems in comprehension. 


\section{Reliability}

Cronbach's $\alpha$ was 0.85 for THA and 0.91 for TKA. Test-retest reliability was measured in all of the subjects and was excellent (ICC $=0.97$ with $95 \%$ CI $0.95-0.98$ for THA; ICC $=0.95$ with $95 \%$ CI $0.92-0.98$ for TKA).

\section{Distribution and floor/ceiling effects}

The adapted HAAS had no significant floor/ceiling effects in both populations (Tables 2 and 3).

\section{Content validity}

The content of the items was considered valid for the evaluation of high-intensity activities. All the questions were judged relevant to investigate high-intensity activities in the populations investigated. The concepts being explored were clearly defined, and described high-intensity activities that might be influenced by THA and TKA.

\section{Construct validity}

Regarding the THA population, there was a moderate correlation between the HAAS-I and NRS $(r=-0.40)$, there was a high correlation between the HAAS-I and WOMAC $(r=-0.68)$ and there were moderate to high correlations between the HAAS-I and SF-36 domains ( $r=0.34$ to 0.63 ). Regarding the TKA population, there was a moderate correlation between the HAAS-I and NRS $(r=-0.77)$, there was a high correlation between the HAAS-I and KOOS subscales $(r=-0.79$ to $r=-0.91)$ and there were low correlations between the HAAS-I and SF-36 domains $(r=0$. 01 to 0.29$)$. Tables 4 and 5 summarize the correlations.

Table 2 Distribution and floor/ceiling effects of HAAS-I and other measures in subjects with total hip arthroplasty

\begin{tabular}{llll}
\hline Outcome measures & $\begin{array}{l}\text { Test } \\
\text { mean (SD) }\end{array}$ & $\begin{array}{l}\text { Re-test } \\
\text { mean (SD) }\end{array}$ & $\begin{array}{l}\text { Floor/ceiling } \\
\text { effects (\%) }\end{array}$ \\
\hline HAAS & $10.50(3.62)$ & $10.52(3.42)$ & $0 / 0(0 \%, 0 \%)$ \\
WOMAC & $17.49(22.40)$ & n.a. & $10 / 0(15 \%, 0 \%)$ \\
WOMAC Pain & $14.68(20.81)$ & n.a. & $21 / 0(31 \%, 0 \%)$ \\
WOMAC Stiffness & $19.22(25.41)$ & n.a. & $32 / 0(48 \%, 0 \%)$ \\
WOMAC ADL & $17.91(22.91)$ & n.a. & $11 / 0(16 \%, 0 \%)$ \\
NRS & $2.01(1.86)$ & n.a. & $1 / 0(2 \%, 0 \%)$ \\
SF-36 Physical Function & $67.23(29.14)$ & n.a. & $0 / 10(0 \%, 15 \%)$ \\
SF-36 Physical Role & $46.30(44.22)$ & n.a. & $27 / 22(40 \%, 33 \%)$ \\
SF-36 Bodily Pain & $62.06(30.00)$ & n.a. & $3 / 17(5 \%, 25 \%)$ \\
SF-36 General health & $32.23(16.90)$ & n.a. & $0 / 0(0 \%, 0 \%)$ \\
SF-36 Vitality & $59.86(17.42)$ & n.a. & $0 / 0(0 \%, 0 \%)$ \\
SF-36 Social function & $72.38(23.80)$ & n.a. & $0 / 12(0 \%, 18 \%)$ \\
SF-36 Emotional Role & $65.17(45.48)$ & n.a. & $20 / 40(30 \%, 60 \%)$ \\
SF-36 Mental Health & $67.00(21.26)$ & n.a. & $0 / 4(0 \%, 6 \%)$ \\
\hline .a. not available, SD standard & & n. & n. \\
\hline
\end{tabular}

n.a. not available, SD standard deviation, ICC intraclass correlation coefficient, $\mathrm{Cl}$ confidence interval, HAAS-I High-Activity Arthroplasty Score (Italian version), NRS numerical rating scale, WOMAC Western Ontario and McMaster University index, SF-36 Short Form Health Survey 36 items
Table 3 Distribution and floor/ceiling effects of HAAS-I and other measures in subjects with total knee arthroplasty

\begin{tabular}{llll}
\hline Outcome measures & $\begin{array}{l}\text { Test } \\
\text { mean (SD) }\end{array}$ & $\begin{array}{l}\text { Re-test } \\
\text { mean (SD) }\end{array}$ & $\begin{array}{l}\text { Floor/ceiling } \\
\text { effects (\%) }\end{array}$ \\
\hline HAAS & $10.50(3.62)$ & $10.52(3.42)$ & $0 / 0(0 \%, 0 \%)$ \\
KOOS Symptoms & $4.40(3.60)$ & n.a. & $10 / 0(16 \%, 0 \%)$ \\
KOOS Pain & $4.72(4.10)$ & n.a. & $13 / 0(21 \%, 0 \%)$ \\
KOOS ADL & $9.00(8.50)$ & n.a. & $15 / 0(25 \%, 0 \%)$ \\
KOOS Sport/Recreation & $8.13(5.03)$ & n.a. & $0 / 0(0 \%, 0 \%)$ \\
KOOS Quality of Life & $3.97(2.54)$ & n.a. & $6 / 0(10 \%, 0 \%)$ \\
NRS & $1.90(1.20)$ & n.a. & $0 / 0(0 \%, 0 \%)$ \\
SF-36 Physical Function & $78.20(18.61)$ & n.a. & $0 / 10(0 \%, 16 \%)$ \\
SF-36 Physical Role & $76.23(32.73)$ & n.a. & $4 / 35(7 \%, 57 \%)$ \\
SF-36 Bodily Pain & $80.57(18.11)$ & n.a. & $0 / 23(0 \%, 38 \%)$ \\
SF-36 General health & $52.21(10.18)$ & n.a. & $0 / 0(0 \%, 0 \%)$ \\
SF-36 Vitality & $69.67(8.60)$ & n.a. & $0 / 0(0 \%, 0 \%)$ \\
SF-36 Social function & $84.01(16.31)$ & n.a. & $0 / 25(0 \%, 41 \%)$ \\
SF-36 Emotional Role & $95.08(21.80)$ & n.a. & $3 / 58(5 \%, 95 \%)$ \\
SF-36 Mental Health & $77.90(9.70)$ & n.a. & $0 / 0(0 \%, 0 \%)$ \\
\hline
\end{tabular}

n.a. not available, $S D$ standard deviation, ICC intraclass correlation coefficient, $\mathrm{Cl}$ confidence interval, HAAS-I High-Activity Arthroplasty Score (Italian version), NRS numerical rating scale, KOOS Knee injury and Osteoarthritis Outcome Scale, SF-36 Short Form Health Survey 36 items

\section{Sensitivity to change}

The MDC was 1.7 for THA and for 1.8 TKA, reflecting the smallest changes in score that are likely to reflect a true change rather than a measurement error.

Table 4 Construct validity. Pearson's correlations between the HAAS-I and WOMAC, NRS and SF-36 in subjects with total hip arthroplasty

\begin{tabular}{lll}
\hline Outcome measures & HAAS-I & $p$ value \\
\hline WOMAC & -0.68 & 0.000 \\
WOMAC Pain & -0.66 & 0.000 \\
WOMAC Stiffness & -0.50 & 0.000 \\
WOMAC Activities of Daily Living & -0.70 & 0.000 \\
NRS & -0.40 & 0.001 \\
SF-36 Physical Function & 0.63 & 0.000 \\
SF-36 Physical Role & 0.46 & 0.000 \\
SF-36 Bodily Pain & 0.47 & 0.000 \\
SF-36 General health & 0.34 & 0.005 \\
SF-36 Vitality & 0.45 & 0.000 \\
SF-36 Social function & 0.42 & 0.000 \\
SF-36 Emotional Role & 0.48 & 0.000 \\
SF-36 Mental Health & 0.35 & 0.003 \\
\hline
\end{tabular}

HAAS-I High-Activity Arthroplasty Score (Italian version), NRS numerical rating scale, WOMAC Western Ontario and McMaster University index, SF-36 Short Form Health Survey 36 items 
Table 5 Construct validity. Pearson's correlations between the HAAS-I and KOOS, NRS and SF-36 in subjects with total knee arthroplasty

\begin{tabular}{lll}
\hline Outcome measures & HAAS-I & $p$ value \\
\hline KOOS Symptoms & -0.80 & 0.000 \\
KOOS Pain & -0.82 & 0.000 \\
KOOS ADL & -0.89 & 0.000 \\
KOOS Sport/Rec & -0.91 & 0.000 \\
KOOS QoL & -0.83 & 0.000 \\
NRS & -0.76 & 0.000 \\
SF-36 Physical Function & 0.28 & 0.032 \\
SF-36 Physical Role & 0.29 & 0.024 \\
SF-36 Bodily Pain & 0.21 & 0.099 \\
SF-36 General health & 0.13 & 0.328 \\
SF-36 Vitality & 0.21 & 0.097 \\
SF-36 Social function & 0.06 & 0.636 \\
SF-36 Emotional Role & 0.26 & 0.026 \\
SF-36 Mental Health & 0.01 & 0.994 \\
\hline
\end{tabular}

HAAS-I High-Activity Arthroplasty Score (Italian version), NRS numerical rating scale, KOOS Knee injury and Osteoarthritis Outcome Scale, SF-36 Short Form Health Survey 36 items

\section{Discussion}

This study describes the adaptation and validation of the HAAS-I in $<65$ years old subjects who underwent primary uncemented THA/TKA because of primary osteoarthritis.

The results of the adaptation process indicate that it was successfully developed following international guidelines. The experts played an important role during the re-evaluation of the process and confirmed the quality of the work done. The on-field text confirmed that the items were easily understandable, leading to a valid measure of another culture's concept of health that allows data comparability and cross-national studies.

The questionnaire was successfully self-administered and seems to be easily applicable in everyday clinical practice.

The HAAS-I was internally consistent, with estimated similar to original findings (THA/TKA: 0.86) and higher than French estimates (TKA: 0.58) [9, 10]. Test-retest reliability was also satisfactory; however, similar estimates were not calculated by the original developers as well as by French researches, and therefore, comparison cannot be conducted [10].

The HAAS-I had no serious floor/ceiling effects, demonstrating its ability to assess wider ranges of disease severity. No effects were also found in the French study for TKA [10].

Construct validity was initially analysed by comparing the HAAS-I with disability scales. The close correlations in both samples suggest that the theoretical constructs of the two measures are very similar. Significant correlations were found by the original developers concerning WOMAC
( $p<0.001$; Spearman coefficients not reported) and Harris Hip Score ( $p<0.003$; Spearman coefficients not reported), while non-significant estimates were for Oxford Knee Scale ( $p<0.017$; Spearman coefficients not reported) [9]; our findings were higher than French results when the HAAS was compared to Oxford Knee Scale (TKA: 0.19) [10]. The moderate to high associations with the NRS suggests that high intensity activities may be linked to the intensity of pain, particularly in subjects with TKA. The original and the French study did not investigate these relationships, and therefore, comparisons cannot be provided [10]. Construct validity was further analysed by relating the HAAS-I with quality of life. Moderate to high correlations were displayed for THA subjects, suggesting that the increase in high-intensity activities is correlated with an increase in perceived quality of life. Surprisingly, poorer correlations were found between the HAAS-I and SF-36 in TKA; the lower level of disability in our sample than previously reported [15] and the low rate of co-morbidities probably explain this unexpected result. Moreover, the distribution analysis showed that the SF-36 had floor and ceiling effects, which suggests uncertainty in the QoL answers. Again, comparison with other studies cannot be conducted.

HAAS-I proved to be also sensitive to change. Given the degree of repeatability, the SEM and SDC were reduced and ensured it could identify changes in the scores exceeding the threshold of instrument noise. At 95\% confidence level, the minimum detectable change indicates that, if an individual shows a change of more than 1.7 points for THA and 1.8 points for TKA after a given intervention, it would not be a measurement error. In other studies, this estimate was never calculated and, therefore, comparisons cannot be made.

This study has some limitations. Firstly, as it was designed cross-sectionally, any significant correlations should not be confused with causal effects. Secondly, the relationships between high-intensity activities and physical tests were not considered because only questionnaires were used. Thirdly, content validity was based on questions that might have prevented neutral responses partially limiting the soundness of our results; the use of open questions in the future is suggested. Finally, a full psychometric assessment including also responsiveness was not conducted, and further studies are therefore recommended.

\section{Conclusions}

The Italian version of the HAAS is reliable, valid and sensitive to change. It can be recommended for clinical and research purposes, and it is expected to improve the high-intensity activities assessment of $<65$ years old subjects after hip and knee arthroplasty. 


\section{Appendix}

\section{High-Activity Arthroplasty Score (HAAS) - Italian version 1.0}

Seleziona il più alto livello funzionale per ognuna delle seguenti categorie:

\section{Camminare (max. 5 punti)}

5 su terreni scoscesi e accidentati per più di un'ora 4 con difficoltà ma senza limiti su terreni accidentati pianeggianti

3 senza limiti su terreni non accidentati pianeggianti

2 su terreni pianeggianti per almeno mezz'ora

1 per brevi distanze senza assistenza (fino a 20 metri)

0 per brevi distanze o pochi passi, mediante ausili

\section{Correre (max. 5 punti)}

4 per più di 5 kilometri

3 blandamente per almeno 5 kilometri

2 attraversare di corsa la strada

1 fare qualche balzo per schivare il traffico, se necessario

0 impossibilitato

\section{Salire le scale (max. 3 punti)}

3 due scalini per volta

2 senza corrimano

1 usando corrimano o bastone

0 impossibilitato

\section{Livello di attività (max. 6 punti)}

6 sport competitivi (tennis in singolo, corsa per più di 10 kilometri, bicicletta per più di 80 kilometri) 5 sport amatoriali (tennis in doppio, sci, corsa per meno di 10 kilometri, sport aerobici ad alto impatto) 4 attività ricreative di alta intensità (passeggiata in collina, attività aerobica a basso impatto, giardinaggio, attività manuali, attività agricole)

3 attività ricreative di media intensità (golf, giardinaggio leggero, attività manuale leggera)

2 attività ricreative di bassa intensità (brevi camminate, bocce)

1 attività all'aperto solo se necessario (camminare per brevi distanze per fare shopping)

0 autonomia solo in ambito domestico

Punteggio totale

\section{Acknowledgements}

The authors wish to thank all of the subjects who took part into the study. Furthermore, we also thank Claudio Secci, Barbara Rocca and Caterina

Albensi for their assistance and comments.

Availability of data and materials

The data and material are available on requests.

\section{Authors' contributions}

MM, AC, LC, GM and PA contributed to the conception and design of the work and the acquisition, analysis and interpretation of data. MM, AC, LC, PA and FM drafted the work for important intellectual content; MM, NM and CF contributed to the conception and design of the work and the acquisition, analysis and interpretation of data and agreed to be accountable for all aspects of the work in ensuring that questions related to the accuracy or integrity of any part of the work are appropriately investigated and resolved. All authors read and reviewed the various versions of the manuscript, and read and approved the final version of the manuscript.

Ethics approval and consent to participate

This cross-sectional study was approved by the Local Ethics Committee of the University of Cagliari and conducted in accordance with ethical and humane principles of research.

\section{Competing interests}

The authors declare that they have no competing interests.

\section{Publisher's Note}

Springer Nature remains neutral with regard to jurisdictional claims in published maps and institutional affiliations.

\section{Author details}

${ }^{1}$ Department of Public Health, Clinical and Molecular Medicine, University of Cagliari, Cagliari, Italy. ${ }^{2}$ Orthopaedic Unit, Department of Surgery, University of Cagliari, Cagliari, Italy. ${ }^{3}$ Department Economics and Business Science, University of Cagliari, Cagliari, Italy. ${ }^{4}$ Scientific Institute of Montescano, Clinical and Scientific Institutes Maugeri, Institute of Care and Research, Montescano, Pavia, Italy. ${ }^{5}$ Department of Musculoskeletal Disorders, University of Salerno School of Medicine and Surgery, Salerno, Italy. ${ }^{6}$ Centre for Sports and Exercise Medicine, Barts and The London School of Medicine and Dentistry, Mile End Hospital, 275 Bancroft Road, London, UK. PPhysical Medicine and Rehabilitation Unit, University of Rome "Tor Vergata", Rome, Italy.

Received: 15 February 2018 Accepted: 27 March 2018

Published online: 11 April 2018

References

1. Vignon E, Conrozier T, Le Graverand M-PH. Advances in radiographic imaging of progression of hip and knee osteoarthritis. J Rheumatol. 2005;32: 1143-5.

2. Bijlsma JW, Berenbaum F, Lafeber FP. Osteoarthritis: an update with relevance for clinical practice. Lancet. 2011;377:2115-26.

3. Torre M, Carrani E, Del Manso M. Progetto Registro Italiano ArtroProtesi. 2015

4. Tegner $Y$, Lysholm J. Rating systems in the evaluation of knee ligament injuries. Clin Orthop. 1985;198:42-9.

5. d'Aubigne RM, Postel M. Functional results of hip arthroplasty with acrylic prosthesis. JBJS. 1954;36:451-75.

6. Charnley J. The long-term results of low-friction arthroplasty of the hip performed as a primary intervention. Bone Jt J. 1972;54:61-76.

7. Banaszkiewicz PA. Traumatic arthritis of the hip after dislocation and acetabular fractures: treatment by mold arthroplasty: an end-result study using a new method of result evaluation. Class Pap Orthop. Springer. 2014:13-7

8. Bellamy N, Buchanan WW, Goldsmith CH, Campbell J, Stitt LW. Validation study of WOMAC: a health status instrument for measuring clinically important patient relevant outcomes to antirheumatic drug therapy in patients with osteoarthritis of the hip or knee. J Rheumatol. 1988;15:1833-40. 
9. Talbot S, Hooper G, Stokes A, Zordan R. Use of a new high-activity arthroplasty score to assess function of young patients with total hip or knee arthroplasty. J Arthroplast. 2010;25:268-73.

10. Diesinger $Y$, Jenny J-Y. Validation of the French version of two on highactivity knee questionnaires. Orthop Traumatol Surg Res. 2014;100:535-8.

11. Beaton DE, Bombardier C, Guillemin F, Ferraz MB. Guidelines for the process of cross-cultural adaptation of self-report measures. Spine. 2000;25:3186-91.

12. Wild D, Grove A, Martin M, Eremenco S, McElroy S, Verjee-Lorenz A, et al. Principles of good practice for the translation and cultural adaptation process for patient-reported outcomes (PRO) measures: report of the ISPOR task force for translation and cultural adaptation. Value Health. 2005;8:94-104

13. Terwee $\mathrm{CB}$, Bot $\mathrm{SD}$, de Boer MR, van der Windt DA, Knol DL, Dekker J, et al. Quality criteria were proposed for measurement properties of health status questionnaires. J Clin Epidemiol. 2007:60:34-42.

14. Salaffi F, Leardini G, Canesi B, Mannoni A, Fioravanti A, Obo CR, et al. Reliability and validity of the Western Ontario and McMaster Universities (WOMAC) Osteoarthritis Index in Italian patients with osteoarthritis of the knee. Osteoarthr Cartil. 2003;11:551-60.

15. Monticone M, Ferrante S, Salvaderi S, Rocca B, Totti V, Foti C, et al. Development of the Italian version of the knee injury and osteoarthritis outcome score for patients with knee injuries: cross-cultural adaptation, dimensionality, reliability, and validity. Osteoarthr Cartil. 2012;20:330-5.

16. Huskisson EC. Measurement of pain. Lancet. 1974;304:1127-31.

17. Apolone G, Mosconi P. The Italian SF-36 health survey: translation, validation and norming. J Clin Epidemiol. 1998;51:1025-36.

\section{Submit your next manuscript to BioMed Central} and we will help you at every step:

- We accept pre-submission inquiries

- Our selector tool helps you to find the most relevant journal

- We provide round the clock customer support

- Convenient online submission

- Thorough peer review

- Inclusion in PubMed and all major indexing services

- Maximum visibility for your research

Submit your manuscript at www.biomedcentral.com/submit 